\title{
Edge preserving orientation adaptive filtering
}

\author{
P. Bakker, L.J. van Vliet, P.W. Verbeek \\ Pattern Recognition Group, \\ Department of Applied Physics, \\ Delft University of Technology, \\ Lorentzweg 1, 2628 CJ, Delft, The Netherlands, \\ \{peterb,lucas,piet\}@ph.tn.tudelft.nl
}

\begin{abstract}
In this paper we describe a new strategy for combining orientation adaptive filtering and edge preserving filtering. The filter adapts to the local orientation and avoids filtering across borders. The local orientation for steering the filter will be estimated in a fixed sized window which never contains two orientation fields. This can be achieved using generalized Kuwahara filtering. This filter selects from a set of fixed sized windows that contain the current pixel, the orientation of the window with the highest anisotropy. We compare our filter strategy with a multi-scale approach. We found that our filter strategy has a lower complexity and yields a constant improvement of the SNR.
\end{abstract}

\section{Introduction}

Noise, which is present in every real world image, hampers manual interpretation by human experts as well as automatic segmentation and analysis by computers. Therefore many image processing techniques are developed to reduce noise. The Wiener filter [8] is the best linear filter but requires a priori knowledge of the spectrum of the noise-free image as well as the spectrum of the noise. Noise in domains without texture can simple be reduced by isotropic smoothing, where the spatial size of the smoothing operator determines the amount of noise reduction. So the size or scale of the domain constitutes the limit to this amount. To optimize the global noise reduction, scale adaptive smoothing can be used. In an oriented texture domain or along individual lines and edges, the noise level can be reduced by applying elongated smoothing operators that adapt to the local orientation. This requires a robust and continuous representation of orientation [1]. Since many natural images can be described as a collection of grey value and oriented texture domains, a scale and orientation adaptive smoothing scheme provides a powerful noise reduction method. Such a scheme can be realized in different ways, i.e. by anisotropic diffusion [10] or steerable filters [2].

Edges between domains are important features for the interpretation of images. However, smoothing operators tend to blur the edges or borders between the different domains. Therefore a filter should be used that reduces the noise but does not degrade the edges, i.e. an edge preserving filter. In a mosaic of domains characterized by grey value, the borders between the domains are characterized by the difference in grey value. This difference can directly be measured in the image. In a mosaic of domains characterized by oriented texture, the borders between the domains are characterized the the difference in local orientation. At an orientation border there are two factors that make filtering more difficult. First, there are locally two dominant orientations, which make it more difficult to estimate the orientation. Secondly, the adaptive filter should not blur across the border.

Examples of edge preserving smoothing can be found in Stereo Matching [4] and anisotropic diffusion [3]. In this paper we will describe a new method for combining edge preserving filtering with orientation adaptive filtering. We present a generalization of the Kuwahara filter for edge preserving smoothing $[5,6]$. The method is tested on both synthetic and natural images. The natural images are seismic images, which are narrow banded and contain layered structures. We will compare our method with a scale adaptive approach [2].

\section{Generalized Kuwahara filtering}

A traditional filter for edge preserving smoothing for images containing grey value domains, is the Kuwahara filter [5]. Kuwahara divided a square symmetric neighborhood 
in four (slightly overlapping) windows, each containing the central pixel, see fig.1a. The central pixel is replaced by the average of the most homogeneous window, i.e. the window with the lowest variance. The combination of filtering (average) and selection (homogeneity) avoids filtering across edges in the image. This filter has been further develop by

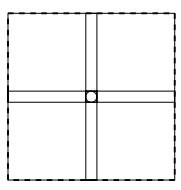

(a)

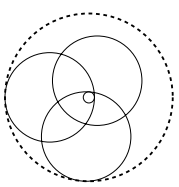

(b)
Figure 1. a) traditional Kuwahara filter, b) generalized Kuwahara filter. The dashed lines bound the neighborhood and the solid lines the windows.

increasing the number of windows to eight and changing the shape of the windows to pentagons and hexagons [6].

Our filter evaluates each fixed size window that contains the current pixel. Each of these windows yields an estimate and a confidence value. The estimate from the window with the highest confidence value is taken as the result. We call this filter the generalized Kuwahara filter and a realization with round windows is depicted in fig. $1 \mathrm{~b}$. Note that the shape of the windows determines the shape of the neighborhood. By applying the generalized Kuwahara filter it is possible to smooth grey value domains and to preserve sharp borders between these domains. A proper representation of the orientation reduces oriented texture domains to grey value domains. Combining the orientation estimation with the generalized Kuwahara filter yields a orientation representation with sharp borders.

\section{Scale adaptive filtering}

A well known general way for dealing with different events at a different scale in a single neighborhood is scalespace. This makes it possible to process each scale separately and thereby provides a way to do scale adaptive filtering. A more computational efficient way is the build an scale pyramid, e.g. octave based difference of low-pass pyramid (DOLP) [9]. Since our images with oriented textures are narrow banded, the frequencies span only two octaves, so the evaluation of three scales should be enough. A filter can be made scale adaptive in a straight forward way by building a DOLP. First apply the filter on each scale. Secondly, the adaptive filtering result is obtained by sum- mation of the filtering results on each scale, see [2]. However, we shall show applying our filtering method on a single scale already yields a good result.

\section{Estimation of steering parameters orienta- tion and anisotropy}

The fundamental proposition on which this work is based, is local one-dimensionality. This means that in textured domains $D$, that constitute the image, contain a single dominant orientation. If we describe the grey values in $D$ with polar coordinates $I(r, \phi)$, we could write

$$
\delta I\left(r, \phi_{0}\right) \gg \delta I\left(r, \phi_{\text {other }}\right), r \in D
$$

where $\delta I$ denotes the change in grey value and $\phi_{0}$ the dominant orientation. We make a distinction between orientation, defined modulo $\pi$, and direction, defined modulo $2 \pi$. Thus two opposite vectors differ in direction but have the same orientation. A robust description of such neighborhood $D$ is given by a tensor representation [1].

$$
\mathbf{T}=\frac{1}{x} \mathbf{x x}^{T}
$$

where $\mathbf{x}$ is a vector along the dominant orientation and $x=$ $\|\mathbf{x}\|$ is the norm of the vector.

\subsection{The Gradient Square Tensor}

The tensor $\mathbf{T}$ from eq. 2 can be implemented in several ways. The general idea is to use a set of directionally selective filters and combine the responses. Examples of directionally selective filters are derivative filters, i.e. first or second order, and quadrature filters [1]. The latter have the advantage to give a response on both edges and lines, but are computationally more expensive. The Gradient Square Tensor (GST) is based on Gaussian first order derivative filters and is given by eq. 3 .

$$
\mathbf{T}_{G S}=\nabla I \nabla I^{T}=\left(\begin{array}{cc}
I_{x}^{2} & I_{x} I_{y} \\
I_{x} I_{y} & I_{y}^{2}
\end{array}\right)
$$

where $I_{x}, I_{y}$ are the Gaussian derivatives in resp. $\mathrm{x}$ and $\mathrm{y}$ direction, in which the Gaussian regularization function has a size $\sigma_{g}$. Since this tensor is a quadratic form the tensor elements may be averaged without having cancellation problems. This averaging is implemented as Gaussian smoothing $\left(\sigma_{T}\right)$ over a window. Applying tensor averaging has three advantages:

1. rapid changes in the orientation estimation due to noise on the gradient vector are suppressed, yielding a smooth result 
2. having only responses on edges no longer constitutes a problem, since on a line the tensor averaging combines the gradients from both slopes, without cancellation of opposite vectors

3. The smoothed tensor allows information about the energy in the dominant and the perpendicular orientation

The local orientation estimation is given by the orientation of the eigenvector corresponding the the largest eigenvalue.

\subsection{Anisotropy estimation}

The certainty of the orientation estimation is proportional to the anisotropy, which describes to what extend one orientation dominates. The anisotropy can be measured from the ratio of the energy in the dominant and perpendicular orientation. We define the anisotropy $A$ as

$$
A=\frac{\lambda_{1}-\lambda_{2}}{\lambda_{1}+\lambda_{2}},
$$

with $\lambda_{1}, \lambda_{2}$ the eigenvalues of the GST. With this definition the anisotropy takes values between 0 and 1 , indicating the range from completely isotrope to perfectly anisotrope.

\subsection{Improved orientation estimation at orienta- tion borders}

Since the GST is based on the assumption that there is locally only one dominant orientation, the corresponding orientation measurement will fail as soon as it crosses an orientation border. The resulting orientation estimation is a weighted average of the two dominant orientations at both sides of the border, causing unsharp or blurred orientation borders. A powerful solution for allowing multiple orientations in one neighborhood in the image, is to add orientation as a new dimension [7]. This can be realized by applying multiple directionally selective filters. The drawback of this approach is the higher computational complexity and will therefor be skipped in this paper.

However, it is possible to allow only one orientations in one neighborhood in the image and also preserve sharp edges. This can be achieved by applying the generalize Kuwahara filter. Calculate the GST in each window and use the anisotropy as the certainty measure. The idea of this method is to prevent that the GST overlaps a border as depicted in fig.2. With the anisotropy measure we can detect an orientation border, since the anisotropy drops if the region over which the GST is smoothed contains an orientation border.

\subsection{Limitations of anisotropy as border detector}

The success of the method described above, depends very much on the estimation of the anisotropy, since this

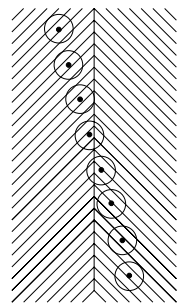

(a)

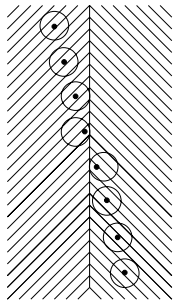

(b)
Figure 2. a) standard GST, b) Improved orientation estimation. The circles denote the regions $\sigma_{t}$ in which the orientation is estimated, the black dots are the positions the result is written to.

parameter is used to locate the borders. It can be derived that:

$$
A \propto \cos (\Delta \phi)
$$

with $A$ the anisotropy and $\Delta \phi$ the difference between the orientation at both sides of the border. As a consequence, for small values of $\Delta \phi$, the anisotropy will decrease only a few percent, e.g. if $\Delta \phi=30 \mathrm{deg}$ the anisotropy will decrease $10 \%$. We experimentally verified this angular dependency and the results are depicted in fig.5. In this

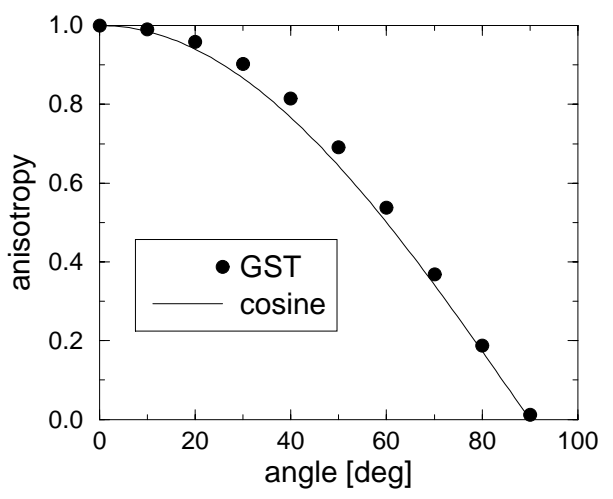

Figure 5. The anisotropy obtained from the GST as a function of the angular difference at an orientation border.

experiment we measured the anisotropy at a border applying the GST with $\sigma_{g}=1.0$ and $\sigma_{T}=5.0$. The measured anisotropy differs slightly from a cosine, as can be seen in fig.5. This is due to the fact that the derivatives are regularized. The reqularization causes the orientations of gradient 


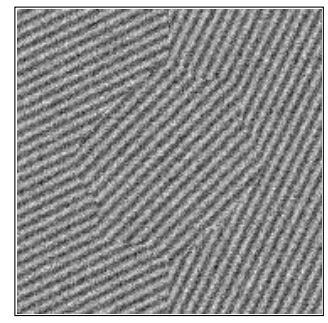

(a)

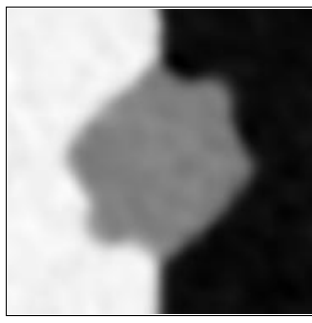

(b)

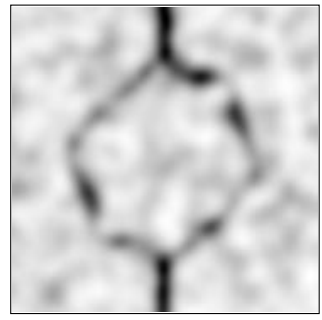

(c)

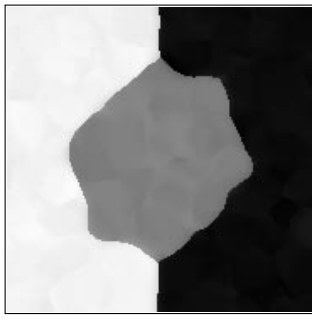

(d)

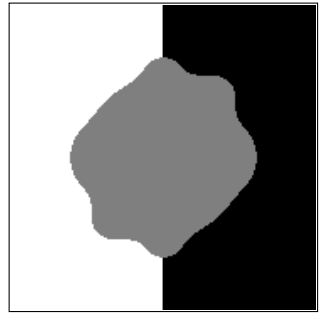

(e)

Figure 3. a) test image, b) orientation estimation GST, c) anisotropy estimation GST, d) improved orientation estimation, e) ground truth for orientation.

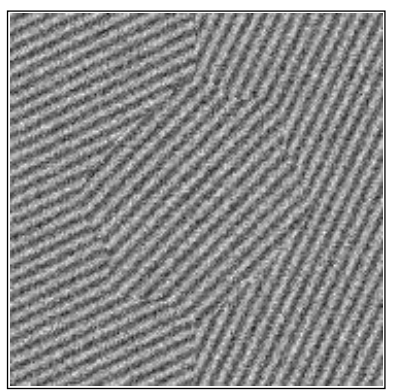

(a)

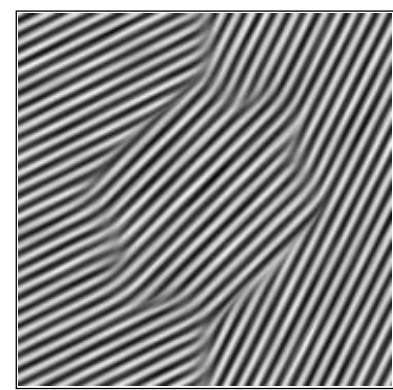

(b)

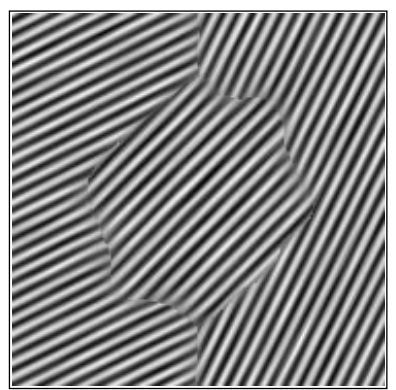

(c)

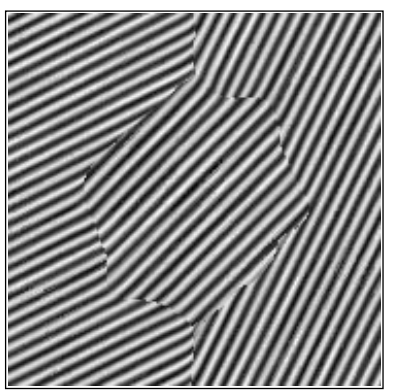

(d)

Figure 4. a) test image, b) adaptive filter steered with orientation from fig.2b, c) same as b but with improved orientation estimation fig.2d, d) same as c but with edge preserving property.

vectors to be distributed between the the two dominant orientations and thereby decreasing the average deviation from the the mean orientation. The anisotropy will increase since it is proportional to the inverse of the average deviation, i.e. the consistency of the orientation.

\section{Adaptive filtering}

Adaptive filtering means that the filter can be controlled by parameters. For example, an orientation adaptive filter given by

$$
F_{\text {adap }}=F(\phi, A)
$$

with $\phi$ the orientation and $A$ the anisotropy, is used in [2]. The idea of this filter is to use the estimated shape and orientation of the tensor $\mathbf{T}$ to adapt the filter behaving in the same way as the signal. The filter can also be made scale adaptive, which is discussed in sec.3. For now we assume that the signal is narrow banded and can be described as single scale signal. Noise reduction in oriented textures can be achieved by steering an elongated filter with the orientation estimation as steering parameter. The orientation of the filter should be perpendicular to to dominant orientation from eq.1. However, sharp domain edges will be blurred by this filter.

\subsection{Edge preserving filtering}

We have shown that it is possible to correctly estimate the orientation near borders, so the filter can correctly be steered along the oriented texture. However, we still have to make sure that the adaptive filter does not overlap borders. This is in fact the same problem we encountered during the orientation estimation. The difference is that we only allow displacement along the layers, as depicted in fig.6. We can solve this problem by applying a one dimensional version of 


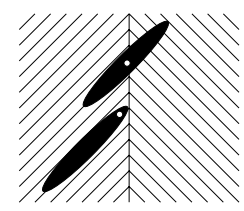

Figure 6. top filter:The orientation adaptive filter is correctly oriented along the layers, but overlaps a border. bottom filter:allowing displacement along the layers

the generalized Kuwahara filter. Since the one dimensional filter is not rotation invariant the orientation can be different for each point in the image, an efficient implementation is not possible. To keep the method computationally inexpensive, we chose to allow only 5 windows evenly spread over the neighborhood.

\section{Experiments and Results}

\subsection{Synthetic images}

To test our method, we created a test image with three textured domains. Each domain consists of a one dimensional sinusoidal signal with a different orientation, see fig.3a. The period of the sinusoidal signal is approximately 6 pixels. Furthermore we added some Gaussian noise, $\mathrm{SNR}=10 \mathrm{db}$.

$$
S N R=20 \log _{10}\left(\frac{A}{\sigma_{n}}\right)
$$

with $A$ the amplitude of the sinusoidal signal and $\sigma_{n}$ the standard deviation of the Gaussian noise. First we applied the GST with $\sigma_{g}=1.0$ and $\sigma_{T}=5.0$, and calculated the orientation and anisotropy, which are depicted in fig.3b and c. The result of the generalized Kuwahara filter combined with the GST is shown in fig.3d, where the Kuwahara neighborhood has a diameter of 11 pixels. The ground truth of the orientation is given in fig.3e for comparison. We applied an orientation adaptive filter that uses the orientation estimation derived from the gradient square tensor as a steering parameter, see fig. $4 \mathrm{~b}$. We also applied the this filter steered with the improved orientation estimation as described in section 4.3 and finally we enhanced this filter with the edge preserving property as described in section 5.1, see fig.4c,d. The filter type we used is the Gaussian filter, with $\sigma=7.0$ in a $29 * 1$ pixel window. A comparison of fig. $4 \mathrm{~b}$ and c, shows that the improvement in orientation estimation yields a clear improvement in filtering near a border. From fig. $4 \mathrm{~d}$ can be seen that the edge preserving method works and yields sharp orientation borders.
To show that our method still gives good results when there is more than one scale present, we repeated the measurement described above on a second test image, see fig.7a. This image has the same domains, only the period of the signals are now 5, 10, 20 pixels. The SNR of this image is $13 \mathrm{db}$. The filter type we used on this image is a Gaussian filter, with $\sigma=6.0$ in a $25^{*} 1$ pixel window.

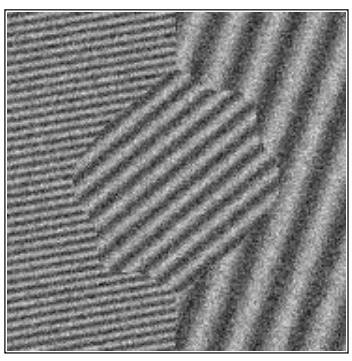

(a)

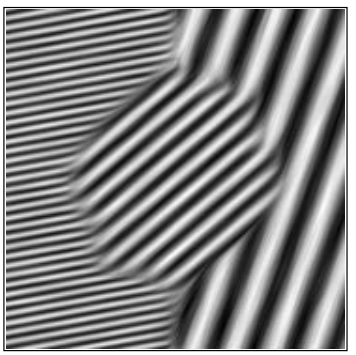

(c)

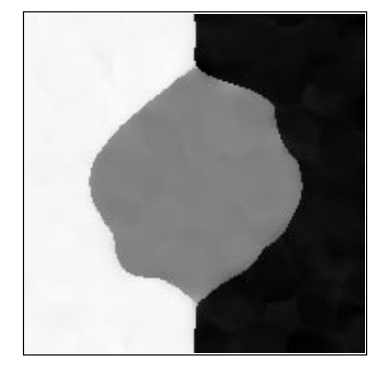

(b)

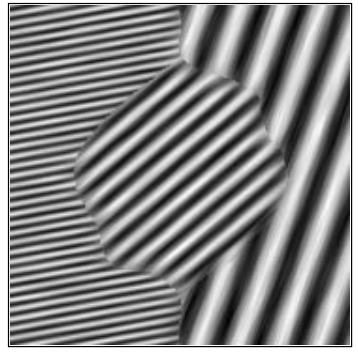

(d)

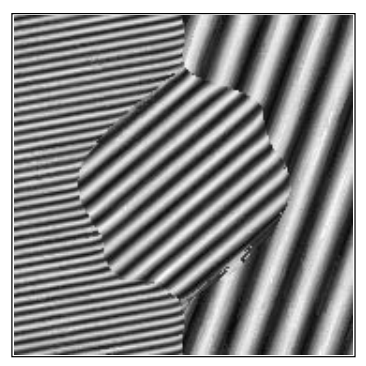

(e)

Figure 7. a) test image, b) Improved orientation estimation, c) adaptive filter steered with orientation estimation from GST, d) same as c but with improved orientation estimation, e) same as $\mathrm{d}$ but with edge preserving property.

\subsection{Natural images}

To test our method on natural images we obtained a seismic image that contains a lot of faults. Again we applied 
our edge-preserving orientation adaptive filter. The filter type we used is the Gaussian filter, with $\sigma=6.0$ in a $25^{*} 1$ pixel window.

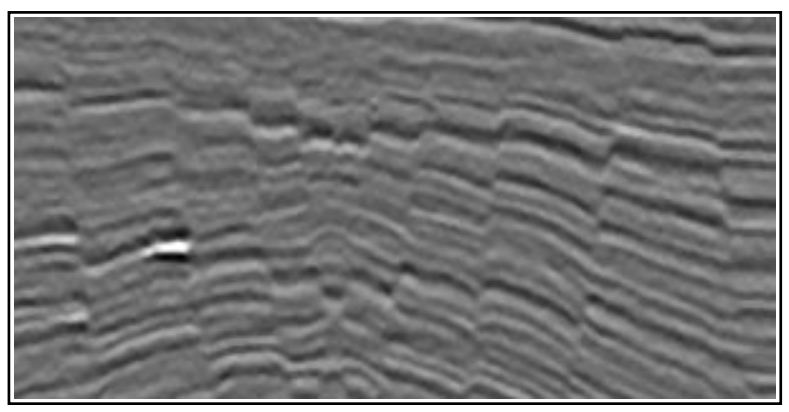

(a)

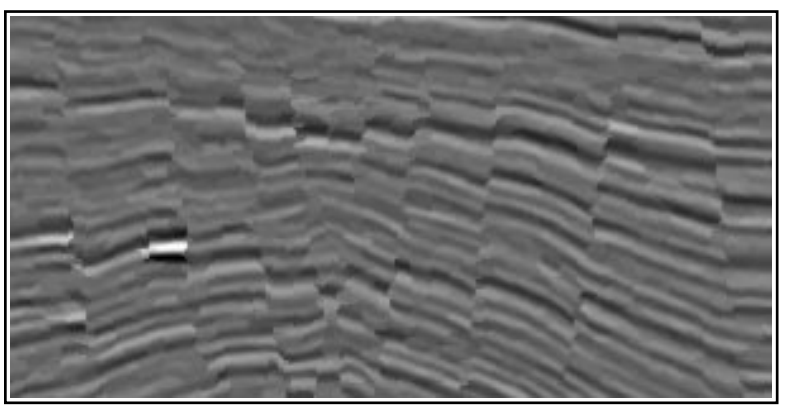

(b)

Figure 8. a) seismic image containing faults, b) edge preserving filtered version of a.

\section{Discussion}

We have shown that it is possible to correctly estimate the orientation near borders by combining the GST with the generalized Kuwahara filter. This makes it possible to correctly steer an orientation adaptive filter in the whole image. Furthermore, this filter is made edge preserving by again applying a one dimensional version of the generalized Kuwahara filter.

Our method yields good results when applied on a single scale in narrow banded image such as seismic images, where narrow banded means that the frequencies present in the image do not span more than two octaves. Compared with multi-scale [2] or orientation space [7] approaches, our method has a low computational complexity. Furthermore, our method gives a constant improvement in the SNR, where as a multi-scale approach would apply smaller filters near the borders which gives less SNR improvement near these borders. The generalized Kuwahara filter may introduce a slight bias in the edge location due to the fact that it uses decentralized orientation estimation.

\section{Acknowledgments}

This research is partly supported by Senter, Agency of the Ministry of Economic Affairs of the Netherlands, the Royal Netherlands Acadamy of Arts and Sciences (KNAW), and the Rolling Grants program of the Foundation for Fundamental Research in Matter (FOM).

\section{References}

[1] Hans Knutsson. Representing Local Structure Using Tensors, The 6th Scandinavian Conference on Image Analysis, Oulu, Finland, pp. 244-251, June 19-22, 1989.

[2] L. Haglund, H. Knutsson, and G.H. Granlund. Scale and Orientation Adaptive Filtering, Proceeding of the 8th Scandinavian Conference on Image Analysis, Volume 2, Tromso, Norway, pp 847-855, May 25-28, 1993.

[3] P. Perona and J. Malik. Scale-Space and Edge Detection Using Anisotropic Diffusion, IEEE Trans. on PAMI, Vol 12. No 7, July 1990.

[4] T. Kanade and M. Okutomi. A Stereo Matching Algorithm with an Adaptive Window: Theory and Experiment IEEE Trans. on PAMI, Vol 16. No 9, September 1994.

[5] M. Kuwahara, K. Hachimura, S. Eiho, and M. Kinoshita. Digital Processing of Biomedical Images., Plenum Press, pp. 187-203, New York, NY, 1976.

[6] Makoto Nagao, and Takashi Matsuyama. Edge Preserving Smoothing, Computer Graphics and Image Processing, Academic Press, pp. 394-407, September 1979.

[7] M. van Ginkel, P.W. Verbeek, L.J. van Vliet. Improved Orientation Selectivity for Orientation Estimation, Proceedings of the 10th Scandinavian Conference on Image Analysis, Lappeenranta, Finland, pp. 533-537, June 9-11, 1997.

[8] W.K. Pratt Generalized Wiener filtering computation techniques. IEEE Trans. Comput., C-21:pp. 297-303, July 1972.

[9] J. Crowley and A. Parker, A representation for shape based on peaks and ridges in difference of low-pass transform. IEEE Trans. on PAMI, (6):pp. 156-169, 1989.

[10] F. Catte, P. Lions, J. Morel and T. Coll, Image selective smoothing and edge detection by nonlinear diffusion. SIAM J. Numer. Anal., Vol. 29, No. 1, pp. 182-193, February 1992 